\title{
Modulation-Aware Energy Balancing in Hierarchical Wireless Sensor Networks ${ }^{1}$
}

\author{
Maryam Soltan, Inkwon Hwang, Massoud Pedram \\ Dept. of Electrical Engineering \\ University of Southern California \\ Los Angeles, CA \\ $\{$ soltan,inkwonhw,pedram\}@usc.edu
}

\begin{abstract}
Wireless sensor networks (WSN) with hierarchical organizations have recently attracted a lot of attention as effective platforms for pervasive computing. With power efficiency and lifetime awareness becoming critical design concerns, a significant amount of research has focused on energy-aware design of different layers of the WSN protocol stack. However, much less has been done in way of incorporating physical layer characteristics at the system deployment stage and analyzing the effects on spatial energy balancing across the network and the resulting overall network lifetime. Our focus is on improving the lifetime of each cluster of sensors in a hierarchical WSN using optimization techniques at the physical layer. Specifically, we study how the location-aware selection of the modulation schemes for sensors can affect their energy efficiency. Furthermore, we show how the energy in the network can be distributed more evenly by proper selection of the modulation schemes for different sensors.
\end{abstract}

Keywords - Power management, Energy aware systems, Wireless, Sensor networks, Modulation.

\section{Introduction}

Lifetime of a Wireless Sensor Network (WSN) is defined as the duration of time after which a certain percentage of the battery-operated sensors exhaust their energy source and drop out of the network. The radio characteristics for each sensor as well as the communication architecture of the WSN directly affects the system lifetime.

Much research has focused on energy-aware design in different layers of the network in order to extend the network lifetime [1-8]. Various WSN architectures have been proposed for different applications. Specifically, there has been a growing interest in hierarchical WSN architectures to reduce the communication burden on the sensor nodes and hence increase the system lifetime [2-4]. One example is a two-tiered network where relays are used to collect and route data [3][4]. More specifically, fixed relays with higher processing capability and higher energy level act as cluster-heads in order to collect events and measurements from a cluster of sensors and forward the data to a central base station.

Although there has been significant research on energy efficient WSNs, much less work has been done on the effects of physical layer characteristics in the deployment architectures and overall system design. Modulation scaling and adaptive modulation techniques are introduced in [6-8] where the modulation level is adjusted to reduce the energy per bit. In [6], the concept of modulation scaling is proposed where at lower data traffic, target bit error rate is increased to reduce the required energy per bit. In [7] the impact of using modulation scaling on packet delivery latency and packet loss is considered. In [8], adaptive modulation is used in a centralized cross-layer approach in order to minimize the total transmission energy consumption of the network by taking into account that every sensor may require a different bit rate according to its application. In these techniques, one common modulation scheme is considered across the network and the level is adjusted to achieve lower required energy per bit. However, scaling to lower energies will result in increase in bit error rate.

In this work, we focus on communication characteristics inside each cluster of a hierarchical WSN. A large number of simple low-power sensor devices are distributed in a field in order to collect sensing data and transmit it towards the assigned relay node. We study the effect of different modulation schemes on the energy consumption of the sensor nodes and demonstrate how proper selection of the modulation scheme can affect the overall distribution of energy consumption in the network and the lifetime of the network.

In Section II, we describe the system architecture and

\footnotetext{
${ }^{1}$ This research was sponsored in part by the National Science Foundation.
} 


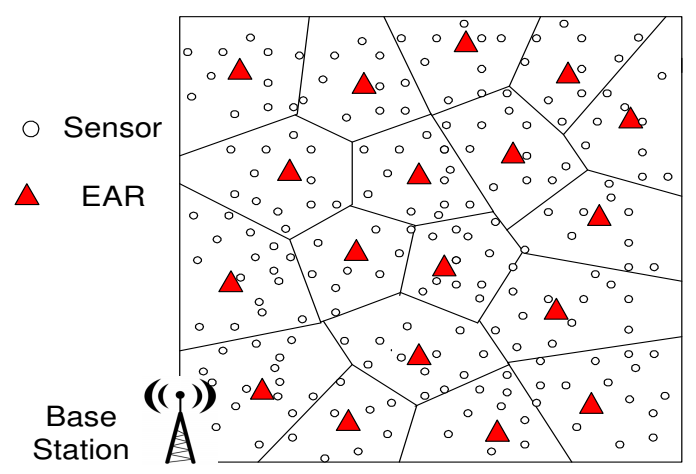

Figure 1: Two-tiered cluster based WSN

our motivation. We then present our analysis in Section III. In Section IV we show our simulation results. Finally, our concluding remarks are presented in Section V.

\section{System description}

A two-tiered WSN consists of event aggregator relay $(E A R)$ nodes and sensor nodes [4]. Sensors form a cluster around each $E A R$ node (cf. Figure 1). The EAR node directly collects data from sensors in its assigned cluster and forwards the data towards the base station. Within a cluster, sensors communicate with the $E A R$ node through different pairs of FDMA/TDMA channels, so there is no co-channel interference between sensors in one cluster.

The network lifetime depends on the lifetime of each cluster. In this study, we try to balance the distribution of energy consumption within each cluster. We assume that $E A R$ nodes have access to much larger energy source than the sensor nodes, so we focus on the energy consumption of the sensors due to data transmission to EAR nodes. By definition, a cluster is at the end of its lifetime when the percentage of live sensors in the cluster falls below a prespecified threshold such that the cluster can no longer satisfy the required quality of sensing for its coverage area. To increase a cluster's lifetime, the energy consumption in each sensor should be reduced.

Energy dissipation due to data transmission is a large percentage of the overall energy consumption within the sensors. Using a log-distance path loss model [11], the required energy per transmitted bit in the $i^{\text {th }}$ sensor node may be written as:

$$
e_{T x}(i)=k_{T x} \cdot E_{b} \cdot\left(\frac{4 \pi d_{e(i), i}}{\lambda_{W}}\right)^{\beta_{e(i), i}}
$$

where $k_{T x}$ is a constant coefficient, which depends on the antenna gain and the output amplifier efficiency of the sensor as well as the receiver Noise Figure of the EAR node. $E_{b}$ is the needed energy per bit at the receiver in order to satisfy a desired maximum bit error rate (BER) requirement. $d_{e(i), i}$ and $\beta_{e(i), i}$ denote the distance and the path loss exponent between sensor $i$ and its assigned EAR node $e(i)$, respectively. $\beta_{e(i), i}$ depends on the environment and is typically between 2 and 5 , whereas $\lambda_{w}$ denotes the signal wavelength.

The distance clearly has a significant impact on the transmission energy dissipation. There have been some studies in WSN to reduce the distance by different routing and assignment techniques [3]. In this paper, we assume that the EAR node placement and sensor assignments have already been performed. Objective is thus to reduce the energy dissipation by optimal selection of the modulation schemes for the pre-placed sensors. For any modulation scheme, the bit error rate can be characterized as a function of $E_{b} / N_{0}$ which is ratio of the energy per bit to the noise power spectral density [9]. For a given bit error rate, there can be a large difference between the required $E_{b} / N_{0}$ of different modulation schemes (cf. Figure 2). For example, to achieve $0.001 \%$ BER, a coherent Binary Phase Shift Keying (BPSK) demodulator requires about 4 dB (i.e., $\sim 2.5$ times) less energy per bit than a non-coherent Binary Frequency Shift Keying (BFSK) demodulator [9][10].

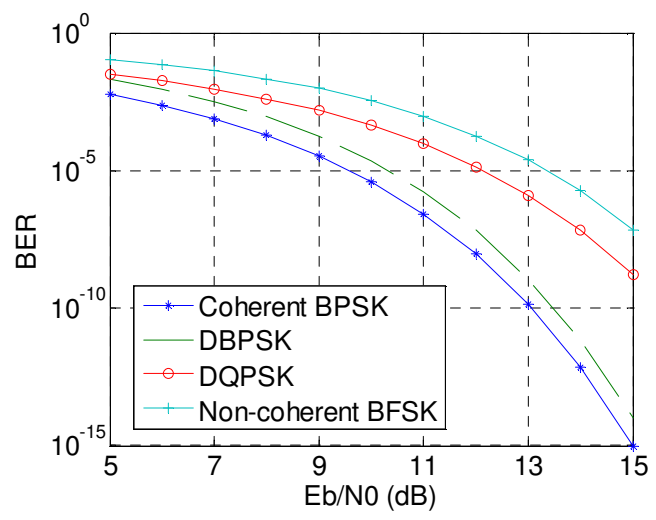

Figure 2: Bit Error Rate vs. $E_{b} / N_{0}$

There is also a trade-off between modulation power efficiency versus the receiver complexity and bandwidth efficiency. Therefore, using the most power efficient modulation for all sensors in the network may not be desirable. In the next section we discuss our approach and analyze the effect of modulation selection on energy distribution.

\section{System analysis and approach}

Knowing the characteristics of different modulation schemes and their $E_{b} / N_{0}$ ratio for a given BER, we investigate how selection of the modulation scheme for various sensors in the cluster at system design and deployment stage can affect the overall energy consumption. We show that by properly selecting modulation schemes for different sensors, we can reduce the average energy consumption and even out the network energy distribution over the coverage area. Note that each sensor may only support one modulation scheme while 


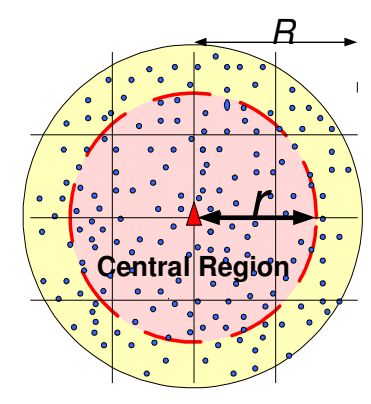

Figure 3: A cluster of sensors with a relay node in center

$E A R$ nodes are assumed to support multiple modulation schemes either via multiple receivers or via a single software-defined configurable receiver.

Let's consider $N$ sensors randomly distributed in an area with radius $R$ where the $E A R$ node is located at the center of the region (cf. Figure 3). For the sensors which are closer to the EAR node, we select Modulation 1 as a less complex modulation scheme that requires larger $E_{b} / N_{0}$. However, for those sensors that are farther away, we select Modulation 2 as a more complex modulation scheme that can achieve the same BER with smaller $E_{b} / N_{0}$. As an example, we may select non-coherent BFSK as Modulation 1 for the sensors within distance $r$ from the EAR node, and coherent BPSK as Modulation 2 for sensors that are farther away. In this example, if Modulation 1 requires $E_{b}$ Jules per bit for a given BER, Modulation 2 will achieve the same BER with about $0.4 E_{b}$ Jules per bit [10].

The average transmission energy per bit for the sensors located within distance $r$ with BFSK (Modulation 1) based on equation (1) is:

$$
\bar{e}_{T x 1}=k_{T x} \cdot E_{b} \cdot\left(\frac{2 \pi r}{\lambda_{w}}\right)^{\beta}
$$

For the farther away nodes which use BPSK (Modulation 2), the average transmission energy per bit is:

$$
\bar{e}_{T x 2}=k_{T x} \cdot 0.4 E_{b} \cdot\left(\frac{4 \pi(r+0.5(R-r))}{\lambda_{w}}\right)^{\beta}
$$

Although Modulation 2 is more complex, utilizing it for the more distant sensors, which have larger path loss and hence higher energy consumption, can result in better spatial balancing of the overall energy in the network. While this shows the effect of modulation selection on the network energy depletion, it should be noted that, in general, distance may not be the only design criterion. Depending on the application and the detailed characteristics of the system, other design criteria such as interference from nearby clusters, fading characteristics, as well as other key modulation attributes in terms of bandwidth efficiency, and system complexity may be considered.

\section{Simulation setup and results}

In this study, we randomly place 2000 sensors using a two-dimensional uniform distribution in a 200x200 $\mathrm{m}^{2}$ field. The sensors are divided into four clusters with $E A R$ nodes placed in the center of each cluster. The packet size is set to 128 bytes, while the average sensing rate is 0.6 $\mathrm{kb} / \mathrm{s}$. The initial battery energy level of each sensor is $2 \mathrm{~kJ}$. There are a total of 500 TDMA/FDMA channels (in 10 frequencies and 50 time slots) for each $E A R$ node so as to avoid any co-channel interference. For each channel, the bit rate is set to $40 \mathrm{~kb} / \mathrm{s}$. The path loss exponent is set to 3 . For our simulation purposes, we consider three different modulation schemes in the area, namely, Differential Quadrature PSK (DQPSK), Differential BPSK (DBPSK), and BPSK. To achieve $0.01 \%$ BER and assuming additive white Gaussian noise (AWGN) channels, the required $E_{b} / N_{0}$ for these modulations can be obtained as $11,9.3$, and $8.4 \mathrm{~dB}$, respectively [10].

Three modulation selection schemes are compared:

- Scheme I (Homogenous Modulation): DQPSK modulation is used in all sensors.

- Scheme II (Homogenous Modulation with Location-Aware Modulation Scaling): DQPSK modulation is used in all sensors, except that three different $E_{b} / N_{0}$ thresholds $(11,9.3,8.4 \mathrm{~dB})$ are selected based on sensor locations.

- Scheme III (Heterogeneous Location-Aware Modulation): DQPSK modulation is used for the centrally located sensors that are within 30 meters from their assigned EAR node. DBPSK modulation is used in more distant sensors that are still within 50 meters from the EAR node. Finally, the rest of the sensors that are located more than 50 meters from the EAR node use the BPSK modulation. This is an example implementation of our proposed location-aware modulation selection methodology.

Figures 4 and 5 depict the spatial distribution of the percentage of remaining energy over initial energy at different simulation times. Figure 4 presents the results for Scheme I. Simulation times correspond to the events where $2 \%$ and $10 \%$ of sensors run out of battery. Figure 5 reports the results for Scheme III at exactly the same simulation times as in Figure 4. These results show exactly how spatial energy balancing is improved.

Table 1 compares the percentage of remaining energy over initial energy as well as the network lifetime for the abovementioned three modulation selection schemes. The results show that our proposed location-aware heterogeneous modulation scheme improves the network lifetime by about $85 \%$ compared to a homogeneous modulation scheme. The lifetime improvement is only about $70 \%$ when a homogeneous modulation scheme with location-aware modulation scaling is employed. This difference is due to the fact that in homogenous modulation 
scaling, there is a larger re-transmission overhead associated with the farther away sensors, which have lower $E_{b} / N_{0}$ threshold, and hence higher BER and packet loss.

These results clearly demonstrate how selecting heterogeneous modulations in the network can balance the energy dissipation in different sensors within a cluster, and thereby, increase the cluster lifetime, and subsequently, the WSN lifetime.

\section{Concluding Remarks}

We studied one aspect of how physical layer attributes can affect the service lifetime of a wireless sensor network. Specifically, we presented a location-aware modulation scheme and showed how the modulation selection can flatten and balance the spatial distribution of energy dissipation over a coverage area in a wireless sensor network. It should be noted that, in general, our proposed location-aware heterogeneous modulation scheme may be implemented in conjunction with other low power techniques in different network layers in order to improve the network lifetime.
TABLE 1: Energy and lifetime results

\begin{tabular}{|c|c|c|c|}
\hline & $\begin{array}{c}\text { Homogenous } \\
\text { modulation } \\
\text { (DQPSK) }\end{array}$ & $\begin{array}{c}\text { Homogenous } \\
\text { modulation } \\
\text { with } \\
\text { modulation } \\
\text { scaling } \\
\text { (DQPSK) }\end{array}$ & $\begin{array}{c}\text { location-Aware } \\
\text { Heterogeneous } \\
\text { modulation } \\
\text { (DQPSK,DBPSK, } \\
\text { and BPSK) }\end{array}$ \\
\hline $\begin{array}{c}\text { Average } \\
\text { remaining } \\
\text { energy over } \\
\text { the initial } \\
\text { energy after } \\
\mathbf{1 0 1 3} \text { days }\end{array}$ & $\begin{array}{c}71 \% \\
(2 \% \text { dead) }\end{array}$ & $\begin{array}{c}81.1 \% \\
(0 \% \text { dead) }\end{array}$ & $\begin{array}{c}82.5 \% \\
(0 \% \text { dead })\end{array}$ \\
\hline $\begin{array}{c}\text { Lifetime } \\
\text { (2\% dead } \\
\text { sensors) }\end{array}$ & $\begin{array}{c}\text { (Days) } \\
\text { (D) }\end{array}$ & $\begin{array}{c}1726 \\
\text { (Days) }\end{array}$ & $\begin{array}{c}1875 \\
\text { (Days) }\end{array}$ \\
\hline
\end{tabular}

\section{References}

[1] V. Raghunathan, C. Schurgers, S. Park, and M. Srivastava,

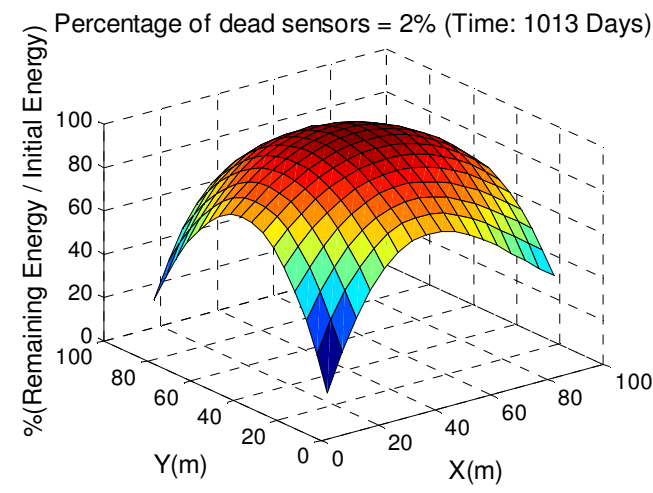

(a)

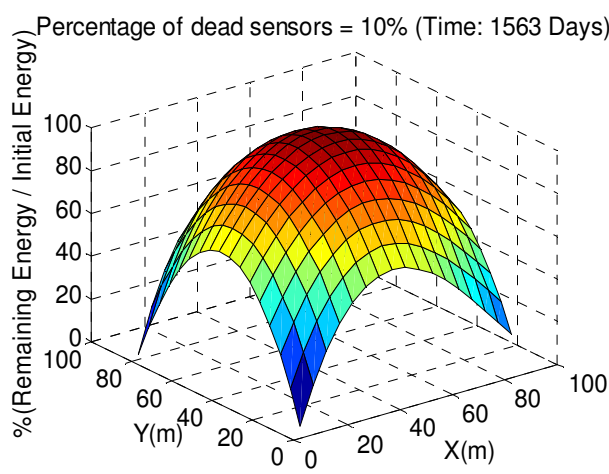

(b)

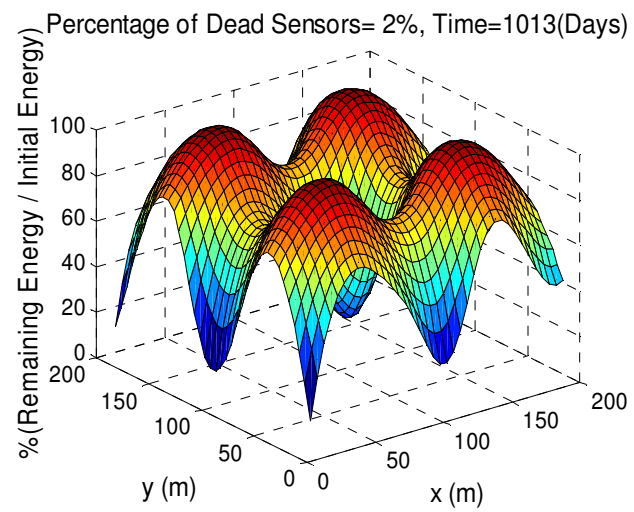

(c)

Figure 4: Spatial distribution of remaining energy over initial energy with homogenous modulations (all sensor nodes use DQPSK) (a) \& (b) One cluster at two different times (c) A network with 4 clusters after $2 \%$ of sensors run out of battery. 


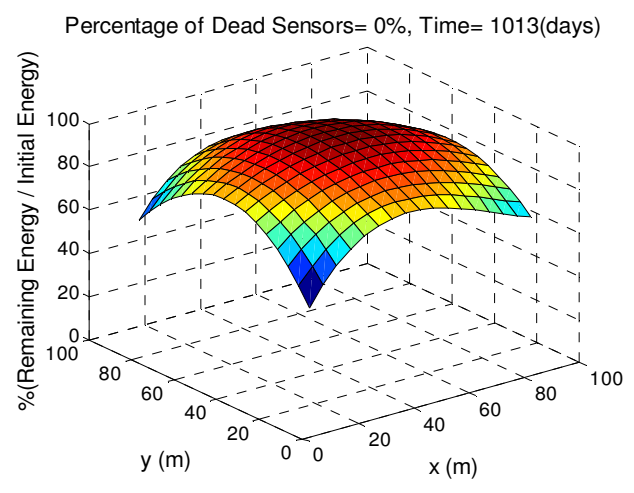

(a)

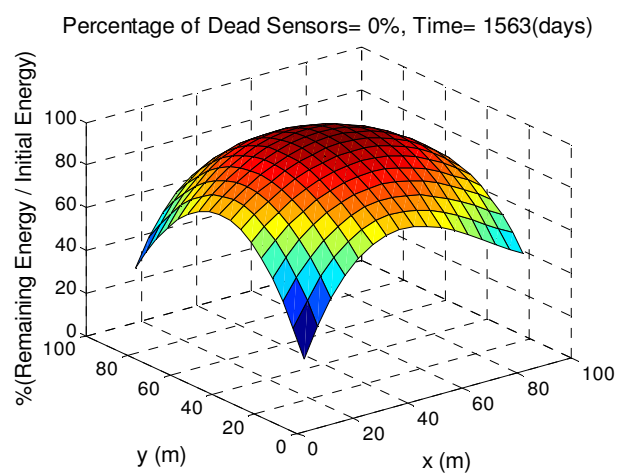

(b)

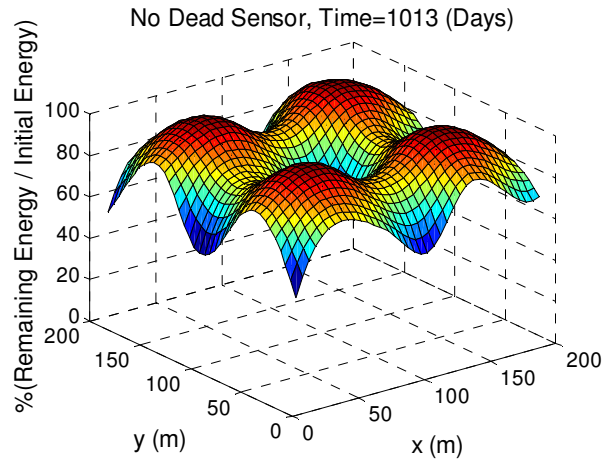

(c)

Figure 5: Spatial distribution of remaining energy over initial energy with location-aware heterogeneous modulation assignments (sensors use DQPSK, DBPSK, or BPSK depending on their distance from the EAR node) -

(a) \& (b) One cluster at two different times (c) A network with 4 clusters after the same time as in Fig. 4-C with no dead sensors

"Energy-Aware wireless microsensor networks," IEEE Signal Processing Magazine, vol. 19, pp. 40-50, March 2002.

[2] V. P. Mhatre, C. Rosenberg, D, Kofman, R. Mazumdar, and N. Shroff, "A minimum cost heterogeneous sensor network with lifetime constraint," IEEE Transactions on Mobile Computing, Vol. 4, No1, pp. 4-15, 2005.

[3] A. Iranli, M. Maleki, and M. Pedram, "Energy-efficient strategies for deployment of a two-level wireless sensor network," Proc. of SLPED, Aug. 2005, pp. 233-238.

[4] M. Soltan, M. Maleki, and M. Pedram, "Lifetime-aware hierarchical wireless sensor network architecture with mobile overlays," Proc. of IEEE Radio and Wireless Symposium, pp. 325-328, Jan. 2007.

[5] H. kwon, T. H. Kim, S. Choi, and B. G. Lee, "A cross-layer strategy for energy-efficient reliable delivery in wireless sensor networks," IEEE Transactions on Wireless Communications, vol. 5, no. 12, pp. 3689-3699, 2006.
[6] C. Schurgers, O. Aberborne, and M. Srivastava, "Modulation scaling for energy-aware communication systems," in ISLPED, pp. 96-99, 2001.

[7] Z. Yang, Y. Yuan, J. He, and W. Chen, "Adaptive modulation scaling scheme for wireless sensor networks," IEICE Transactions on Communications, pp. 882-889, 2005.

[8] J. J. E. Garzás, C. B. Calzón, and G Armada, “An EnergyEfficient Adaptive Modulation Suitable for Wireless Sensor Networks with SER and Throughput Constraints," EURASIP Journal on Wireless Communications and Networking, 2007.

[9] J. G Proakis., Digital Communications, McGraw-Hill Inc., Third Edition, 1995.

[10] Xiong, F., Digital Modulation Techniques, Artech House, 2000.

[11] Rapparport, T. S. , Wireless Communications, Principles and Practice, Prentice Hall, 1996. 